\title{
A Matrix-Vector Construction of the Algebra of Complex Numbers
}

\section{Jeffrey UhImann}

Department of Electrical Engineering and Computer Science, University of Missouri, Columbia, USA

Email address:

uhlmannj@missouri.edu

\section{To cite this article:}

Jeffrey Uhlmann. A Matrix-Vector Construction of the Algebra of Complex Numbers. Applied and Computational Mathematics. Vol. 8, No. 1, 2019, pp. 1-2. doi: 10.11648/j.acm.20190801.11

Received: December 10, 2018; Accepted: January 2, 2019; Published: January 30, 2019

\begin{abstract}
The aim of this paper is to describe an alternative way to think about the algebra of complex numbers that may be of pedagogical value for introducing related concepts such as linear transformations and convolutions. The method is to define a fixed linear transformation of complex numbers represented in vector form so that products can be evaluated elementwise in the transformed space. The principal results are concrete demonstrations that this can in fact be accomplished.
\end{abstract}

Keywords: Algebras, Complex Numbers, Convolutions, Hypercomplex Algebras, Mathematics Education, Vector Spaces

\section{Introduction}

We present an alternative construction of the algebra of complex numbers that potentially provides insights not offered by conventional expositions of the topic. Specifically, we show how complex numbers can be represented as vectors with arithmetic operators defined in terms of a special convolution-like linear transformation. Although the principal goal of the exercise is pedagogical, the approach provides potentially interesting generalizations to higher dimensions.

\section{A Matrix Construction of the Algebra Complex Numbers}

The simplest generalization from the ordinary algebra of scalar variables to multiple dimensions is to define a vector of scalar elements and apply operators componentwise, e.g., addition and multiplication of 2-dimensional vectors would be:

$$
\begin{gathered}
{\left[\begin{array}{l}
a \\
b
\end{array}\right] \oplus\left[\begin{array}{l}
c \\
d
\end{array}\right] \doteq\left[\begin{array}{l}
a \\
b
\end{array}\right]+\left[\begin{array}{l}
c \\
d
\end{array}\right]=\left[\begin{array}{l}
a+c \\
b+d
\end{array}\right]} \\
{\left[\begin{array}{l}
a \\
b
\end{array}\right] \otimes\left[\begin{array}{l}
c \\
d
\end{array}\right] \doteq\left[\begin{array}{l}
a \\
b
\end{array}\right] \circ\left[\begin{array}{l}
c \\
d
\end{array}\right]=\left[\begin{array}{l}
a c \\
b d
\end{array}\right]}
\end{gathered}
$$

where "o" is the standard elementwise (Hadamard) product operator $[2,3]$. In a sense this algebra is just a parallel application of ordinary scalar addition and multiplication to a collection of scalar variables. In other words, the vectors are just arbitrary collections of scalars that for all practical purposes are treated independently of each other.

The most familiar algebra over intrinsically 2-dimensional objects is that of complex variables, $a+b i$, where $i$ is the socalled imaginary unit: $i=\sqrt{-1}$. Although the addition of two complex numbers is analogous to an elementwise operator, multiplication definitely is not [1]:

$$
(a+b i) \cdot(c+d i) \rightarrow(a c-b d)+(a d+b c) i
$$

Thus, in the case of addition we could represent complex numbers as vectors:

$$
a+b i \equiv\left[\begin{array}{l}
a \\
b
\end{array}\right]
$$

and apply simple elementwise vector addition to obtain the correct vector parameterization of the sum of two complex numbers. Of course elementwise multiplication won't yield the correct result under this interpretation, but suppose there existed a matrix $\mathrm{T}$ that could be applied to transform vectors to a space where the product can be applied elementwise and then apply the inverse matrix $\mathrm{T}^{-1}$ to the result. For example, if vectors $u$ and $v$ are defined as

\footnotetext{
1 Alternatively, one could think of the transformation as decorrelating the components to form an orthogonal basis.
} 


$$
u=\left[\begin{array}{l}
a \\
b
\end{array}\right] \text { and } v=\left[\begin{array}{l}
c \\
d
\end{array}\right]
$$

then the product would be evaluated as

$$
\mathrm{T}^{-1}((\mathrm{~T} u) \circ(\mathrm{T} v)) \rightarrow\left[\begin{array}{l}
a c-b d \\
a d+b c
\end{array}\right]
$$

Does there exist such a matrix T? Yes, it can be verified that the following scaled unitary matrix will do the trick:

$$
\mathrm{T}=\left[\begin{array}{cc}
1 & -i \\
1 & i
\end{array}\right]
$$

The details of this matrix, or how precisely it does what we need, is not really important. What is important is that it can be verified to evaluate the product of complex numbers represented in the form of simple vectors. Specifically, multiplication cannot be performed elementwise because the product of complex numbers involves a "mixing" of the the parameters/elements. What the matrix $\mathrm{T}$ provides is a means for transforming vectors to a space in which corresponding vector elements can be multiplied independently. The result can then be transformed back via $\mathrm{T}^{-1}$, and it is this transformation process where the mixing and then unmixing of the parameters occurs ${ }^{1}$.

Instead of working with real vectors we could have retained the imaginary unit and defined our vectors as

$$
u=\left[\begin{array}{c}
a \\
b i
\end{array}\right] \text { and } v=\left[\begin{array}{c}
c \\
d i
\end{array}\right]
$$

for which the necessary transformation can be verified to be the real matrix

$$
\left[\begin{array}{cc}
1 & -1 \\
1 & 1
\end{array}\right]
$$

It might be tempting to wonder if multiplication of the 4dimensional generalization of complex numbers, i.e., quaternions $[4,5]$, could be effected in a similar way. The answer is no because the elementwise product is commutative while quaternion multiplication is not. However, an infinitude of commutative convolutional algebras can be defined in any number of dimensions simply by choosing a particular nonsingular matrix $\mathrm{T}$ and defining the addition and multiplication operators in the way we've already considered:

$$
\begin{gathered}
u \oplus v \doteq u+v \\
u \otimes v \doteq \mathrm{T}^{-1}((\mathrm{~T} u) \circ(\mathrm{T} v))
\end{gathered}
$$

Of course the properties of the product will depend on the choice of matrix, e.g., instead of the Euclidean norm of the product being the product of the norms of the multiplicands which holds in the case of complex numbers - there will be different properties determined by the specific choice of $\mathrm{T}$.

\section{Conclusion}

We have considered a representation of complex numbers in the form of simple 2-dimensional vectors. What was observed is that addition could be performed elementwise while multiplication could only be performed elementwise in a different $2 \mathrm{~d}$ space. This motivated the derivation and use of a transformation to that space to allow the product to be evaluated elementwise before transforming back to the original vector space. Although this approach is primarily of pedagogical interest, it is hoped that the exercise has provided interesting insights that may lead to useful generalizations.

\section{References}

[1] Lars Ahlfors, Complex Analysis (3rd ed.), McGraw-Hill, 1979.

[2] J. Hadamard, "Resolution d'une question relative aux determinants," Bulletin des Sciences Mathematiques Series, 2 (17), pp. 240-246, 1893.

[3] R. A. Horn and C. R. Johnson, Matrix Analysis, Cambridge University Press, 1990.

[4] W. Hamilton, ed., Elements of Quaternions, London (UK), 1866.

[5] I. L. Kantor and A. S. Solodvnikov, Hypercomplex Numbers: An Elementary Introduction to Algebras, New York: SpringerVerlag, 1989. 